\title{
Correspondence
}

\author{
Editor: Greg Wilkinson
}

Contents: MAOIs and narcotic analgesics/Comparison of electrical measurements on constant voltage and constant current ECT machines/Selfpoisoning/Late paraphrenia/Comparative trial of a new antidepressant/The symbolic significance of Munchausen's syndrome/Down-regulation of postsynaptic serotonin receptors as a mechanism for clomipramine-induced anorgasmia/Abnormal intestinal permeability: an aetiological factor in chronic psychiatric disorders?/Mental handicap and doubleblind trial design/Gilles de la Tourette's syndrome in Down's syndrome/Onset of schizophrenia in men and women/Chronic schizophrenia and long-term hospitalisation/DST in apathetic hyperthyroidism/ Catatonic signs in schizophrenia/Chronic depression in general practice/John Langdon Down.

\section{MAOIs and Narcotic Analgesics}

SIR: Brown \& Linter's review (Journal, August 1987, $151,210-212$ ) was a welcome review of the confused area of interactions between MAOIs and narcotic analgesics, and provided some useful practical guidelines.

The interaction between dextromethorphan and the MAOIs is, however, contentious. The authors refer to a letter by Rivers \& Horner (1970) concerning a patient receiving phenelzine $(60 \mathrm{mg} /$ day $)$ who died after consuming $2 \mathrm{oz}$ (about $55 \mathrm{ml}$ if standard fluid ounces are assumed) of a cough mixture containing dextromethorphan. This is open to question, as the dose of dextromethorphan was not stated, nor the other ingredients of the preparation, and nor could the death of the patient definitely be attributed to an interaction (Ascione, 1976). A second letter in the same journal also reporting a dextromethorphan-MAOI interaction death (Shamsie \& Barriga, 1971 ) is similarly doubtful, as the preparation also contained phenylephrine, a drug known to interact adversely with MAOIs. Animal experiments (Sinclair, 1973) show an interaction between dextromethorphan and MAOIs. However, the fatal doses used in the rabbits were very high, and a similar reaction can also be produced with imipramine in rabbits. The strict relevance of these animal studies must thus be questioned, since imipramine and MAOIs are used in clinical practice together, albeit with great care, without reported incidents.

The statement that dextromethorphan is absolutely contra-indicated with MAOIs must thus remain open to considerable doubt.

STEPHEN BAZIRE

Hellesdon Hospital

Norwich NR6 $5 B E$

\section{References}

Ascione, F. J. (1976) Information sources-separating fact from fiction. Drug Therapy, 6, 43-45.

RIVERS, N. \& HORNER B. (1970). Possible lethal reaction between Nardil and dextromethorphan. Canadian Medical Association Journal, 103, 85 .

Shamsie, S. J. \& Barriga, C. (1971) The hazards of use of monoamine oxidase inhibitors in disturbed adolescents. Canadian Medical Association Journal, 104, 715.

SinClaIR, J. G. (1973) Dextromethorphan-monoamine oxidase inhibitor interaction in rabbits. Journal of Pharmacy and Pharmacology, 25, 803-808.

\section{Comparison of Electrical Measurements on Constant Voltage and Constant Current ECT Machines}

SIR: When calculating the energy produced by the constant voltage ECT machine (Journal, August $1987,151,244-247)$, we used a formula based on the assumption of a $50 \%$ modified sine wave. The manufacturers (Ectron) state that the machine produces " $55 \%$ sine waveforms". Examination of the curves produced by the machine in our trial indicates that the waveform is more accurately described as a $60 \%$ modified sine wave. In the latter case we have underestimated the energy produced by the constant voltage machine by approximately $40 \%$. Consequently, all quoted values for energy produced by the constant voltage machine should be multiplied by 1.39 . The conclusions in the paper are unaffected by the above.

I am grateful to Dr Barrington of Herrison Hospital, Dorchester, for pointing out this error.

\section{Monklands District General Hospital \\ Monkscourt Avenue \\ Airdrie ML6 0JS}

R. RAILTON 